\title{
Der innere Orient des barocken Trauerspiels
}

Als ein Verfahren der Identitätsbildung durch Abgrenzung wird der Orientalismus vor allem mit dem Zeitalter der europäischen Kolonialexpansion im 19. und in der ersten Hälfte des 20. Jahrhunderts in Verbindung gebracht. Er hat aber eine Vorgeschichte, die weit in die Epoche der Türkenkriege zurückreicht. Um diesen Orientalismus avant la lettre soll es im Folgenden gehen, genauer: um seine Ausprägungen im schlesischen barocken Trauerspiel. Die These ist, dass auch die beiden wichtigsten Vertreter dieser literarischen Gattung, Andreas Gryphius (1616-1664) und Daniel Casper von Lohenstein (1635-1683), Edward Saids Orientalismus-Buch rezipiert und in ihren Dramen verarbeitet haben. Allerdings ist der Orient des Barock eine machtpolitisch und phantasmengeschichtlich komplizierte Angelegenheit, und noch viel mehr der ,innere Orient' des Barock. Es sind deshalb zunächst die historischen Rahmenbedingungen zu umreißen, bevor etwas detaillierter auf die literarischen Werke eingegangen werden kann.

Bis in die Frühe Neuzeit hinein war das christliche Abendland sehr viel kleiner als Europa. Seit der 1492 abgeschlossenen Reconquista gehörte zwar die iberische Halbinsel wieder dazu, aber Griechenland, die Wiege Europas, und große Teile Südosteuropas unterstanden dem osmanischen Reich, das bestrebt war, über Ungarn weiter zu expandieren, und sogar Wien bedrohte. 1683, im sogenannten Türkenjahr, standen die Türken vor Wien und wurden erst von da an allmählich zurückgedrängt (Großer Türkenkrieg 1683-1699). Auch das Mittelmeer war nach dem Fall von Konstantinopel 1453 zu großen Teilen nicht christlich, sondern islamisch, zumal sich das osmanische Reich über den gesamten nordafrikanischen Raum erstreckte.

Dieser im kulturellen Gedächtnis der Jetztzeit nur erstaunlich schwach präsente Sachverhalt hat erheblich an der Formation eines militärisch-politischen Begriffs von Europa mitgewirkt und prägt ihm bis heute seinen Stempel auf. Felix Konrad schreibt dazu:

In den Schriften und Reden italienischer Humanisten der Mitte des 15. Jahrhunderts wurde „Europa“ als eine Einheit entworfen, die in scharfem Gegensatz zu den Türken stand. Die Rückführung der Türken auf das Barbarentum der Skythen, die Umdeutung des ersten Kreuzzugs in ein europäisches Unternehmen, das der Verteidigung gegen Barbaren diente, sowie die Übertragung von politisch-moralischen Verpflich- 
tungen auf die Großeinheit „Europa“ als „Vaterland“ in Türkenkriegsreden konstruierten Europa als eine Identität stiftende Einheit. Damit wurde „Europa“ eine Bedeutung zugewiesen, die der Begriff zuvor nie gehabt hatte, und dies in Opposition zu den Türken. ${ }^{1}$

Das religionshistorische Großereignis des 16. Jahrhunderts, die Reformation, spielt sich vor diesem Hintergrund ab. Luther verstand es, die Türken in sein apokalyptisches Weltbild einzufügen; er teilte ihnen die Rolle einer "Geißel Gottes“ zu. ${ }^{2}$ Generell war, so noch einmal Konrad,

[d]as Islambild im Zeitalter der Renaissance und der Reformation [...] von der Türkengefahr geprägt. Es transportierte einerseits althergebrachte religiöse Topoi (der Islam als Häresie, als Macht des Antichristen), andererseits ethnische Stereotypen (die Türken als Barbaren), die ebenfalls in einer langen Tradition standen. Immer erschienen die Türken als das fundamental Andere und als eine existentielle Gefahr für das Eigene. $^{3}$

Die Propaganda gegen die Türken vereinigte Reformatoren und Gegenreformatoren. Dabei wurden Stereotypen verwendet, die zu den Konstanten kultureller Mobilmachung zu gehören scheinen, etwa das Stereotyp von der bedrohten Jungfräulichkeit der eigenen Töchter beziehungsweise des eigenen Kollektivs. Von Abraham a Santa Clara, einem der volkstümlichen Prediger des 17. Jahrhunderts, ist im Türkenjahr 1683 der Appell überliefert: „auf, auf ihr Christen, jetzt gehet es Gottes Ehr, jetzt gehet es das Erbgut der Braut Christi an".4

Das deutsche Barock hatte also nicht nur seinen Orient - in Gestalt von sehr nahe gekommenen Kriegsgegnern, weitläufigen Handelsbeziehungen, fremden Gütern und Beschreibungen von Reisen in ferne Länder -, sondern auch seinen "Orientalismus", im Sinn von Edward Said verstanden als die Selbstdefinition Europas respektive des Westens im Licht einer Alteritätserfahrung, die im fremden Spiegel des Orients eine eigene Identität findet und stabilisiert. Und wie bei Said ist ,Orient' dabei weniger eine präzise geographische Angabe als ein diffuser Sammelbegriff. Er umfasst die fernöstlichen Hochkulturen (China, Indien) ebenso wie Persien und den arabischen Raum unter Einschluss der gesamten afrikanischen Mittelmeerküste, die sich ja, geographisch gesehen, im Süden Europas befindet.

1 Felix Konrad, „Von der ,Türkengefahr“ zu Exotismus und Orientalismus: Der Islam als Antithese Europas (1453-1914)?", in: Europäische Geschichte Online (EGO), Mainz, 3.12.2010 (http://www. ieg-ego.eu/konradf-2010-de), S. 8.

2 Ebd., S. 14.

3 Ebd., S. 17.

4 Zit. n. Günter Barudio, Das Zeitalter des Absolutismus und der Aufklärung, Frankfurt/M.: FischerTaschenbuch-Verl. 1981 (= Fischer Weltgeschichte Bd. 25), S. 275. Die von Barudio angegebene Belegstelle ist allerdings nicht verifizierbar. 
Auch im barocken Trauerspiel fließen die Vorstellungen des Orients und Afrikas ineinander. Auf Gryphius' Catharina von Georgien, das von der Gefangenschaft und dem grausamen Ende einer christlichen Königin am Hof des persischen Schah Abbas I. handelt und damit einen historischen Stoff des 17. Jahrhunderts zum Gegenstand hat, wird noch zurückzukommen sein. Lohenstein, der jüngere der beiden Dramatiker, verfasst zwei afrikanische und zwei türkische Trauerspiele letztere, Ibrahim Bassa und Ibrahim Sultan betitelt, stehen am Anfang und am Ende seiner literarischen Laufbahn, schließen sein Gesamtwerk also gleichsam in sich ein. So verschieden die Stoffe im Einzelnen sind, die im Übrigen in ganz unterschiedlichen Epochen spielen, weisen sie in ihrer Charakterisierung der nichteuropäischen, ,exotischen' Akteure doch gemeinsame Merkmale auf. Je weiter man sich vom jeweiligen Zentrum Europas entfernt, desto ungestümer, heißblütiger, unbedachter, irrationaler, weiblicher und am Ende in politischer wie persönlicher Hinsicht selbstmörderischer verhalten sich die Protagonisten. Selbst wenn sie physisch überleben wie der persische Schah in Gryphius'Trauerspiel, müssen sie sich doch im Nachgang der eigentlichen Handlung noch einmal in heller Verzweiflung auf der Bühne zeigen und ewige Höllenstrafen ankündigen lassen. Extrapoliert man aus dem Schicksal der Orientalen/Afrikaner das Schicksal ihrer Völker und Länder, dann ist die Welt jenseits Europas einem ebenso sicheren wie selbstverschuldeten Untergang geweiht.

All dies würde den Rückschluss nahelegen, dass die Ereignisse auf exotischen Schauplätzen den deutschen Dramatikern als besonders geeignete Objekte für Alteritätskonstruktionen (und, in einem dialektischen Wechselverhältnis, dem gegenübergestellte Identitätsbestimmungen) erschienen. Sie wären dann Lehrstücke für einen eher simpel verstandenen Orientalismus in Saidscher Manier. Indessen gibt es schon vor dem genaueren Blick in die Texte zwei Gründe, einer solchen einfachen Darstellung zu misstrauen. Der erste ist literarischer Art: Denn es ist auffällig, dass den Barockdichtern in ganz ähnlicher Weise Episoden aus der römischen Geschichte - genauer: aus der Kaiserzeit - als Faszinationsstoff gedient haben. Insbesondere Nero, der Fama nach der blutrünstigste unter den römischen Kaisern, hat es den Dramatikern und Librettisten des 17. Jahrhunderts angetan. Aber es hieße doch den Begriff des Orientalismus überdehnen, wollte man ihn umstandslos auch auf das antike Rom übertragen, zumal sich ja das Heilige Römische Reich Deutscher Nation in der politischen Nachfolge des Imperium Romanum sah.

Der zweite Grund, weshalb man die Orientdarstellungen im Barockdrama nicht in ein zu schlicht binäres Schema von Alterität und Identität hineinpressen sollte, ist machtpolitischer Natur und führt in die äußerst unübersichtlichen Verhältnisse der Epoche des Dreißigjährigen Krieges. Sowohl Gryphius als auch Lohenstein bekleideten wichtige Ämter als Syndici der schlesischen Landstände beziehungsweise der Stadt Breslau, das heißt als Rechtsvertreter schlesischer Protestanten gegenüber einem immer stärker zentralistisch und absolutistisch orientierten Haus Habsburg, 
das zudem die Rekatholisierung der unter seiner Vormacht stehenden Länder betrieb. Sie hatten die heikle Aufgabe, dem österreichischen Kaiser nach den Wirren der Konfessionskriege einen Restbestand an politischer und verwaltungstechnischjuridischer Autonomie, vor allem jedoch an religiöser Freiheit abzutrotzen. Lohenstein war deshalb 1675 in diplomatischer Mission in Wien, aber auch seine literarischen Werke sind durch Vorreden, Widmungen und allegorische Reyen in den politischen Kontext seiner Zeit eingebunden. ${ }^{5}$ Vor diesem Hintergrund darf man annehmen, dass sich die Sympathie von schlesischen Juristendichtern wie Gryphius und Lohenstein mit einer nach vollständiger politisch-militärischer Kontrolle strebenden Zentralmacht in Grenzen hielt, während sie umgekehrt mit der prekären Lage von Kleinmächten am Rand des Machtraumes von Imperien bestens vertraut waren. Wenn sie nun genau solche Kämpfe an der Peripherie in ihren Dramen durchspielen, allerdings versetzt in eine je nach historiographischer Vorlage mehr oder minder , orientalisierte ${ }^{\iota}$ Umgebung, dann sind die Zuschreibungen von Identität und Alterität nicht so eindeutig, wie es auf den ersten Blick scheinen mag.

Hier lohnt sich ein Seitenblick auf Catharina von Georgien. Die politische Situation des Kleinkönigtums, über das Catharina vor ihrer Gefangenschaft herrschte und das sich im Einflussbereich dreier Großmächte befand (Persien, Russland, osmanisches Reich), weist gewisse Parallelen zur Situation des protestantischen Schlesien auf, wie auch in der Forschung bemerkt wurde. ${ }^{6}$ Spinnt man die Analogie fort, dann rückt der österreichische Kaiser in die Funktionsstelle des persischen Despoten, der sowohl seine persönlichen Gelüste als auch seine machtpolitischen Aspirationen mit äußerster Brutalität zu befriedigen sucht. Die Schilderung höfischer Ränke und Exzesse, die in die topische Klage über das weltverfallene Treiben der Residenzen einmündet, erscheint wie überhaupt die ganze barocktypische Vanitas-Rhetorik in einem anderen Licht, wenn man zugleich im Auge behält, mit welcher Beharrlichkeit die schlesischen Dichterjuristen zur gleichen Zeit daran arbeiten, einmal errungene ständische beziehungsweise städtische Freiheiten vor Übergriffen des Souveräns zu bewahren.

5 Grundlegend zu diesem Aspekt von Lohensteins Wirken: Adalbert Wichert, Literatur, Rhetorik und Jurisprudenz im 17. Jahrhundert: Daniel Casper von Lohenstein und sein Werk; eine exemplarische Studie, Tübingen: Niemeyer 1991.

6 Elida Maria Szarota, Geschichte, Politik und Gesellschaft im Drama des 17. Jahrhunderts, Bern/München: Francke 1976, S. 130ff. - Zur politischen Lesbarkeit dieses Dramas vgl. Lothar Bornscheuer, „Diskurs-Synkretismus im Zerfall der politischen Theologie. Zur Tragödienpoetik der Gryphschen Trauerspiele" in: H. Feger (Hg.), Studien zur Literatur des 17. Jahrhunderts. Gedenkschrift für Gerhard Spellerberg (1937-1996), Amsterdam/Atlanta: Rodopi 1997 (Chloe 27), S. 489-529. - Albrecht Koschorke, „Das Begehren des Souveräns. Gryphius' Catharina von Georgien“, in: D. Weidner (Hg.), Figuren des Europäischen. Kulturgeschichtliche Perspektiven, München: Fink 2006, S. 149-162. - Torsten W. Leine, „Das Martyrium als Politikum. Religiöse Inszenierung eines politischen Geschehens in Andreas Gryphius' Catharina von Georgien“, in: DVjs 84 (2010), S. 160175. - Vgl. auch die wichtige Studie von Chenxi Tang, „International Legal Order and Baroque Tragic Play: Andreas Gryphius' Catharina von Georgien“, in: DVjs 88:2 (2014), S. 141-71. 
Das gilt entsprechend für die ,Orientalisierung' der Herrscherfiguren, wie sie sich in einigen der Dramen findet. Dieses darstellerische Verfahren macht von dem vielleicht zentralen Orient-Klischee des Barockzeitalters Gebrauch, dass nämlich türkische Herrscher besonders unbeherrscht und gewalttätig seien. Solche Etikettierungen dienen aber zugleich als Waffe in den staatspolitischen Auseinandersetzungen innerhalb von Europa. Als , türkisch“ im Sinn von ausschweifend, verderbt, launisch und machtbesessen ließen sich im Zeitalter des Absolutismus durchaus auch die eigenen Fürsten und Könige denunzieren. ,Sultanismus' war eine politische Kampfvokabel, die dem jeweiligen Herrscher eine unrechtmäßige Überdehnung seiner Befugnisse unterstellte und damit, nach monarchomachischer Lehre, seinen Sturz und in letzter Konsequenz seine Tötung rechtfertigte. Wenn Sultan Ibrahim, der Titelheld von Lohensteins letztem Drama, gleich in der ersten Abhandlung in das Schlafgemach der Witwe seines Bruders einbricht und sich dann, in seiner sexuellen Gier zugleich lenkbar wie ein Kind, ersatzweise ein anderes, jungfräuliches Opfer in Gestalt der Tochter des Mufti zuführen lässt, dann ist er damit in überdeutlicher Weise als heilloser Tyrann markiert. Denn das Prinzip des Tyrannen ist die Übertretung, wobei die Schändung der Frauen metonymisch dafür einsteht, die Ehre, das Recht und den Besitz der Untertanen insgesamt zu missachten. In den Worten von Jean Bodin, der mit seinen Six livres de la République von 1576 eines der Grundbücher der neuzeitlichen Souveränitätslehre verfasst hat:

Das vornehmste Unterscheidungsmerkmal des Königs vom Tyrannen besteht nun aber darin, daß der König sich den Gesetzen der Natur beugt, während der Tyrann sie mit Füßen tritt, und darin, daß der eine Frömmigkeit, Gerechtigkeit und Treue hochhält, während der andere sich weder um Gott, noch um Gesetz und Treue schert. Der König läßt nichts unversucht, wenn er meint, es könnte dem gemeinen Wohl und dem Schutz der Untertanen dienen. Der Tyrann tut alles nur um seines eigenen Vorteils, der Rache oder des Vergnügens willen. [...] Ein König achtet die Ehre der anständigen Frau, dem Tyrannen hingegen bedeutet ihre Schändung Triumph.?

Nun sind die Trauerspiele der beiden schlesischen Dichter nichts weniger als antiabsolutistische Polemiken oder offen gegen den Kaiser und das Haus Habsburg gerichtete Traktate. Im Gegenteil, gerade Lohenstein flicht in seine Werke kunstfertige Huldigungen an das österreichische Kaiserhaus ein. Das führt zu eigentümlichen Dissonanzen, etwa wenn der zweite Reyen des afrikanischen Trauerspiels Sophonisbe, das vom jähen Umschlag der Leidenschaft, von Treulosigkeit, Ehebruch und Verrat handelt, sich in eine Art Hochzeitscarmen für Kaiser Leopold I. und seine erste Gemahlin, die spanische Infantin Margarita Teresa, im Jahr 1666

7 Jean Bodin, Sechs Bücher über den Staat, Buch I-III, München: Beck 1981, Buch IV-VI, München: Beck 1986. 2. Buch, Kap. 4, S. 353. 
verwandelt. $^{8}$ Ähnliches gilt für Ibrahim Sultan, Lohensteins letztes Drama, das mit dem Sturz Ibrahim I. (1615-1648) einen zeitgenössischen Stoff behandelt und damit insofern leichtes Spiel hat, als dieser Sultan wohl tatsächlich unfähig und dekadent, womöglich sogar wahnsinnig war. Das Stück ist dem Kaiser anlässlich seiner zweiten Hochzeit mit Claudia Felicitas 1673 gewidmet und stellt „die zu unserer Zeit sichtbare Verfinsterung eines Oßmannischen Mohnden“, veranschaulicht an den wüsten Umtrieben des Sultans, der kaiserlichen Ehe als einer Vermählung von Tugend und Keuschheit entgegen. ${ }^{9}$

Aus heutiger Sicht wären sowohl Sophonisbe als auch Ibrahim Sultan ein denkbar ungeeigneter Stoff für eine Hochzeitsgabe. Wenn man jedoch den grässlichen und exzessiven Charakter des Dramengeschehens nicht einfach Lohensteins spätbarock übersteigerter Manier zurechnen möchte, dann könnte gerade in der Kontrastwirkung die entscheidende Botschaft liegen. Deren manifester Text würde lauten: So seid ihr nicht, ich führe euch eine exotische, sich in ihren Lüsten und Lastern verausgabende Gegenwelt vor, um eure Tugend umso heller erstrahlen zu lassen. Aber im Subtext wäre durchaus die Warnung vor der Übertretung jener roten Linie zu lesen, die den Monarchen vom Tyrannen trennt: Nehmt euch in Acht, dass ihr nicht auch zu Afrikanern und Orientalen werdet! Gemäß der absolutistischen Staatslehre kann man den Souverän politisch nicht binden, weil es keine Instanz geben darf, die über ihm steht; dies würde nur wieder zu Zerwürfnis und Bürgerkrieg führen. Infolgedessen muss man ihm auf dem sanfteren Weg von Fürstenspiegeln, Opern und Dramen den rechten Weg weisen. Der Literatur kommt hier also die Funktion einer weichen Steuerung zu, indem die poetische Gerechtigkeit, die sie walten lässt, an die Existenz eines höheren, über der Fürstenwillkür stehenden Rechtes gemahnt. ${ }^{10}$ Um diese Mahnung überhaupt an den Mann zu bringen, so ließe sich mutmaßen, camoufliert der diplomatisch erfahrene Dramendichter Lohenstein sie als Ergebenheitsadresse eines sich verbeugenden Untertanen. Seine Dichtung bestünde dann in einem nicht unriskanten Balanceakt zwischen einer Demonstration der grässlichen Folgen von Fürstenwillkür und einer an den mächtigsten Fürsten im deutschsprachigen Raum gerichteten poetischen Hommage.

Die Sache ist aber insofern noch komplizierter, als in Lohensteins afrikanischen Trauerspielen Sophonisbe und Cleopatra die Position der fremdländischen Titelheldinnen ja rein machtpolitisch der prekären Lage gerade der Kleinmächte entspricht, in deren Namen die Dramendichter als Rechtsbevollmächtigte agieren. Beide Stücke, in wesentlichen Elementen strukturgleich gebaut, sind mit der Sensibilität von machttechnisch erfahrenen Akteuren geschrieben, die nur zu genau wissen, dass

8 Daniel Casper von Lohenstein, Sophonisbe. Trauerspiel, hg. von R. Tarot, Stuttgart: Reclam 1996, Schluss der zweiten Abhandlung, S. 59.

9 Daniel Casper von Lohenstein, Ibrahim Sultan: in: ders., Afrikanische Trauerspiele, hg. von K. G. Just, Stuttgart: Hiersemann 1957, S. 89-265, Zuschrifft', S. 102.

10 Eingehend dazu: Albrecht Koschorke u.a. (Hg.), Der fiktive Staat. Konstruktionen des politischen Körpers in der Geschichte Europas, Frankfurt/M.: Fischer 2007. Teil III: Der Körper des Souveräns, S. $103 \mathrm{ff}$. 
politische Rationalität eine Frage der Perspektive und der Zuschreibung ist. Sie lassen sich demgemäß als Analysen der Mechanismen imperialer Machtausdehnung lesen, denen die eben noch unabhängig scheinenden Herrscherinnen an der Peripherie des Imperiums zum Opfer fallen. Zu Beginn der Handlung sind ihre Länder zwar bedroht und in kriegerische, diplomatische und heiratspolitische Abwehrgefechte verwickelt, aber immerhin autonom; am Ende finden sie sich als Provinzen in das Römische Reich einverleibt. Letztlich ergeht es auch den Verbündeten der Römer nicht besser, die zwar als Herrscher über die umstrittenen Länder eingesetzt werden und insofern erfolgreicher agieren, aber nur um den Preis, fortan Vasallen zu sein. So oder so ist Widerstand gegen den Hegemon zwecklos und erscheint in einem höchst unvernünftigen Licht.

Man muss hier zwischen der Textoberfläche, die einer topischen Ordnung gehorcht, und dem ,Kleingedruckten' der auf diesem Weg kommunizierten Machtanalyse sehr genau unterscheiden. Oberflächlich betrachtet, ergehen sich die betreffenden Trauerspiele in Orientalismus-Klischees, die wirkungsvoll mit Geschlechterstereotypen verquickt sind. Tugend und Laster sind auf dieser Ebene klar verteilt. Sowohl Sophonisbe als auch Cleopatra scheinen unfähig zu sein, ihre sexuelle Begehrlichkeit zu bezähmen und von ihrer politischen Rolle zu trennen. Sie werden als in hohem Maß promiskuitiv dargestellt, verlieben sich ohne Anlaufzeit in den jeweiligen Sieger, dem sie sich auf der Stelle hingeben wollen, und tragen keine Bedenken, ihren bisherigen Gatten zu verraten. Dadurch ziehen sie die anderen nichtrömischen Protagonisten in ein überhitztes Spiel von Liebesallianzen, Treuebrüchen, Rachegelüsten und opportunistischen Frontwechseln hinein, die allein den Römern zuspielen. Ganz anders die römische Seite: Hier finden sich keine Frauen, nur Militärs. Wenn es auch römische Männer gibt, die sich von der Hitzigkeit der orientalischen Afrikaner anstecken lassen - etwa die Figur des Laelius in Sophonisbe -, so besteht ihre dramaturgische Funktion doch allein darin, den Einzug des Befehlshabers vorzubereiten. Und diese Zentralfigur, die gemäß den Auftrittsregeln von Herrscherdramen lange auf sich warten lässt, ist von weiblicher Verführungskunst nicht zu erweichen. Ihre idealtypische Darstellung findet sie in der Gestalt des römischen Heerführers Scipio in Sophonisbe: Er verkörpert nicht nur die Tugend der constantia, die auch dem neostoizistisch geprägten Barock so teuer war, sondern ist geradezu ein wandelndes Prinzip der Homosozialität: Freundschaft unter Männern gilt alles, Frauen dagegen sind unverlässlich, weshalb man sich von ihnen fernhalten sollte. ${ }^{11}$

Nimmt man die Fremdzuschreibungen in den Dramen für bare Münze, dann ist die Sachlage klar: Einer männlich dominierten Römerwelt, die ein Monopol auf

11 Vgl. Scipios Rede in Lohenstein, Sophonishe, vierte Abhandlung, Verse 261ff., die dazu eine ganze Reihe von denkwürdigen Sentenzen enthält, etwa: „Ein' Handvoll Ehre wigt zwölf Kisten Wollust weg." (Vers 295). - Näheres dazu in meinem noch unpublizierten Vortrag „The Fate of Sophonisbe. Female Eroticism and Male Friendship in 17th Century Drama“, University of Chicago, April 2008. 
politische Rationalität zu halten scheint, steht eine weibliche oder effeminierte Sphäre irrationalen Verhaltens gegenüber, die zudem klar rassische Merkmale trägt. Auch die Ägypter werden als „Mohren“ bezeichnet, ebenso wie Cleopatra, deren historisches Vorbild aber doch aus einer makedonischen Herrscherfamilie stammt ${ }^{12}$. Wenn Augustus der ägyptischen Königin ein galantes Kompliment machen will, heißt es in bester kolonialer Attitüde: „[...] Das stoltze Rom glaub't nicht: / Daß dieses braune Land so weisse Mohren hege; / Noch; daß ein edler Geist hier eine Seele rege. "13 Im Übrigen erfährt man, dass Afrikaner lügen, nur zum Dienen bestimmt, wild und nicht zähmbar sind. ${ }^{14}$ Auch die imperialen Grundrechenarten finden Anwendung, wenn es bei einer militärischen Lagebesprechung heißt, dass Caesar einen römischen Bürger „mehr als tausend Mohren schätzt “. ${ }^{15}$ Viel Raum wird darauf verwendet, dem Römer Antonius, der sich mit der ägyptischen Königin Cleopatra einließ, sein doppeltes Fehlverhalten vorzurechnen: erstens den Verstoß gegen die heterosexuelle Norm, das heißt die Rollentrennung der Geschlechter - „Den Männern kommt der Thron / den Weibern Bettgewand“"16 _, zweitens den damit verknüpften Verstoß gegen eine ethnische Norm, denn man mag sich mit Nichtrömerinnen vergnügen, soll sie aber nicht heiraten. ${ }^{17}$ Von Antonius als einem politischen wie erotischen Überläufer muss deshalb der Nachweis verlangt werden, „Daß sein Gemütte nicht zu sehr Egyptisch sei“.${ }^{18}$ Der Gesandte, der diese Rede führt, legt sich sogar die barocktypische Vergänglichkeitstopik zugunsten der römischen Sichtweise zurecht: „allein’ erweg't: daß einer Frauen hold / Nur schlipffrig Zucker sei / der Zepter aber Gold“" ${ }^{19}$

Indessen lässt sich die dramatische Handlung auch von der Seite der Subalternen her ausbuchstabieren. Aus dieser Sicht verfolgen Sophonisbe und Cleopatra ein einziges, sehr nachvollziehbares Ziel, dem sie alle anderen Rücksichten unterordnen: nicht in die Hände der Römer zu fallen und sich nicht in einem Triumphzug als Kriegsbeute vorführen lassen zu müssen. Auch ihre erotischen Manöver dienen einem politischen Kalkül, nämlich Notallianzen zu stiften, um nicht nur Leben und Status der eigenen individuellen Person, sondern auch den Fortbestand ihrer jeweiligen Königreiche zu sichern. Sie sind deutbar als Verzweiflungstaten im Belagerungszustand. Das jähe Umspringen der Präferenzen ist weniger eine Folge charakterlicher Unbeständigkeit als der wechselnden Nachrichtenlage, hat also weniger mit entfesselter Leidenschaft als mit Opportunitätserwägungen in Momenten höchster Gefahr zu tun. Die beiden Herrscherinnen setzen ihre erotischen Reize als Mittel ein, um sich in der Welt männlicher Krieger zu behaupten. Cleo-

12 Daniel Casper von Lohenstein, Cleopatra. Trauerspiel. Text der Erstfassung von 1661, Stuttgart: Reclam 1998. Erste Abhandlung, Vers 50 und passim.

13 Ebd., IV, $472 \mathrm{ff}$.

14 Ebd., IV, S. 101.

15 Ebd., IV, Vers 95.

16 Ebd., I, Vers 445.

17 Ebd., Vers $473 \mathrm{ff}$.

18 Ebd., Vers 537.

19 Ebd., Vers $597 f$. 
patra will den siegreichen Augustus nicht deshalb verführen, weil sie „das geile Weib“ ist, als das er sie denunzier ${ }^{20}$, sondern weil sie sich und ihr Reich nicht „mit Schimpf und Schmach in Abgrund stürtzen" lassen will. ${ }^{21}$ Das rettet sie nicht davor, am Ende einer regelrechten misogynen Treibjagd ${ }^{22}$ den Freitod wählen zu müssen. Als Sterbende tritt sie vollends aus der Ordnung des politischen Diskurses heraus und wird zu einer abjekten Kreatur. Dasselbe Schicksal ereilt die sie umringenden Frauen, die ihrem Beispiel folgen. Einer der Römer kommentiert den Todeskampf ihrer Vertrauten Charmium mit den Worten: „Schaut! wie der Wurm sich krümmt! si rechelt / si vergeht." ${ }^{23}$

Bei alledem ist die dramatische Konvention in Rechnung zu stellen, dass Liebe und Hass zwischen den Königen Chiffren für politische Allianzen beziehungsweise Feindschaften sind. Wer zwischen Liebe und Hass hin- und herschwankt, scheint für das politische Geschäft ungeeignet; doch zunächst ist das nur ein Beleg dafür, dass er im militärisch-diplomatischen Kräftespiel die schlechteren Karten hat. Die Mächtigen können leicht Stoiker sein und fühlen sich mit einer höheren Vernunft im Bunde, während die peripheren Akteure vom Gang der Entwicklung ins Unrecht gesetzt werden, was immer sie tun. Je mehr sie sich in ihrer Ohnmacht verstricken, desto leichter ist es, sie zu Wesen niederen Ranges herabzusetzen. Seit jeher ist es das Merkmal imperialer Rhetorik, die Widersacher an den Rändern des eigenen Herrschaftsbereichs als irrationale Akteure oder schlichtweg als Delinquenten erscheinen zu lassen. Mit anderen Worten, die Vertreter des Imperiums sprechen sich ein Monopol auf legitimes Machthandeln zu. Die Sterbereden jedoch, die Lohenstein seine Heldinnen halten lässt, durchkreuzen dieses Monopol exemplarisch die Rede der Cleopatra, die sich für den Freitod zurüstet:

August hat Marck und Bein uns ausgesogen /

Den väterlichen Thron durch schlimmes Recht entzogen /

Des Ptolomaeus Schatz durch Schelm-Stück an sich bracht /

Doch ruht sein Ehrgeitz nicht. Er ist nun auch bedacht /

Nach Rom ins Sigs-Gepräng zum Schau-Spiel uns zuführen.

Dis ist es / was wir nur noch haben zuverlihren. ${ }^{24}$

Aus den vorstehenden Ausführungen sollte deutlich geworden sein, dass der Orient im Barock wandert. In Gestalt der Türken befindet er sich in einem näherrückenden Außen, das teils zu Appellen an eine gemeinsame europäisch-christliche Identität, teils zu taktischen Vorteilen im Ränkespiel der rivalisierenden europäischen

20 Ebd., IV, Vers 241.

21 Ebd., V, Vers 61.

22 Vgl, ebd., IV, Vers $211 \mathrm{ff}$.

23 Vgl. V, Vers 254.

$24 \mathrm{~V}$, Vers $62 \mathrm{ff}$. 
Länder genutzt wird. Doch die Attribute des Orientalischen können auch nach innen gewendet werden und in einem indirekten rhetorischen Manöver den Sittenverfall der europäischen Höfe, die Maßlosigkeit der Herrscher und ihr despotisches Gebahren markieren. Das Orientalische wäre dann nur eine Art wüster Übersteigerung der Weltverfallenheit und Heillosigkeit der politischen Sphäre, wie sie die Barockdichtung wieder und wieder beklagt; es hätte dabei eine ähnliche Verfremdungsfunktion wie die Darstellung der römischen Kaiser, die nicht einmal vor dem Mord ihrer nächsten Verwandten zurückschrecken, um sich an der Macht zu halten. ${ }^{25}$ Schließlich lassen sich aber auch Parallelen zwischen orientalisierenden Settings wie in Lohensteins afrikanischen Trauerspielen und den kleinen Herrschaften im Alten Reich ziehen. Das wäre der ,innerste 'Orient als Gegenstand einer Machtanalyse, die das erzwungene Rationalitätsgefälle zwischen imperialen Zentren und Peripherien untersucht. In Gryphius' Trauerspiel Catharina von Georgien steht diese Konfrontation insofern noch unter vertrauten religiösen Vorzeichen, als Catharina eine christliche Königin, ihr Widersacher Chach Abas, also der persische Schah, dagegen als prototypischer mohammedanischer Despot gekennzeichnet ist. In dieser Konstellation kann Catharina ihr Leiden als christliches Martyrium interpretieren. Lohensteins Trauerspiele dagegen weisen die Rolle der subalternen Heldin heidnischen Frauen zu. Auch diese Frauen übrigens machen in ihrer Zurüstung zu einem erzwungenen Freitod von der Bildsprache des Martyriums Gebrauch. Mit anderen Worten, auch die für die Geschichte des Christentums so zentrale Figur des Märtyrers überschreitet im barocken Trauerspiel die Grenze zwischen Innen und Außen, zwischen ,uns' und ,ihnen'. Sie wird zu einer konfessionsübergreifend einsetzbaren Option politischer Rhetorik - einer Inanspruchnahme des Heiligen durch diejenigen Protagonisten an den Rändern der Macht, die dem imperialen Rationalitätsprinzip nicht anders zu begegnen vermögen. ${ }^{26}$

25 So in Lohensteins Agrippina, einem Drama, das manche Züge mit Cleopatra teilt.

26 Weitere Überlegungen zur politischen Funktion des Martyriums im Barock in: Albrecht Koschorke, „Märtyrer/Tyrann“, in: N. Kaminski (Hg.), Andreas Gryphius. Leben - Werk - Wirkung. Berlin: de Gruyter 2014 (im Erscheinen). 